\title{
Assessment of Airway Distribution of Transnasal Solutions in Mice by PET/CT Imaging
}

\author{
ML Soto-Montenegro, ${ }^{1}$ L Conejero, ${ }^{2} \mathrm{JJ}$ Vaquero, ${ }^{1} \mathrm{ML}$ Baeza, ${ }^{2} \mathrm{JM}$ Zubeldia, ${ }^{2} \mathrm{M}$ Desco ${ }^{1}$ \\ ${ }^{1}$ Unidad de Medicina y Cirugía Experimental, Hospital General Universitario Gregorio Marañón, Dr. Esquerdo, 46, 28007, Madrid, Spain \\ ${ }^{2}$ Servicio de Alergia, Hospital General Universitario Gregorio Marañón, Madrid, Spain
}

\begin{abstract}
Purpose: Transnasal administration is one of the most common routes for allergen challenge in mouse models of airway diseases. Although this technique is widely used, neither the amount of allergen that reaches the lung nor its airway distribution has been well established. We used positron emission tomography (PET) and computed tomography (CT) to examine the anatomical distribution of a solution containing a tracer immediately after transnasal delivery and to determine the possible influence of age and administered volume.

Procedures: Forty-six female BALB/c mice were divided into three groups according to instillation volume and age: (A) $15 \mu \mathrm{l}, 8-10$ weeks old $(N=10)$, (B) $30 \mu \mathrm{l}, 8-10$ weeks old $(N=$ $20)$, and $(C) 30 \mu l, 32$ weeks old $(N=16)$. Anesthetized animals underwent a dynamic scan in a dedicated small-animal PET scanner immediately after transnasal administration of a solution containing ${ }^{18} \mathrm{FDG}$. Regions of interest were used to obtain quantitative data. Animals were also imaged with a small-animal CT scanner to obtain complementary anatomical information.

Results: Mean \pm SD $(5.69 \pm 4.51 \%)$ of the solution administered reached the lungs in group A, $41.84 \pm 8.03 \%$ in group B, and $36.65 \pm 16.15 \%$ in group C. A comparable percentage was delivered to the left and right lungs in all the groups. Analysis of variance revealed a significant difference between the groups in the proportion of the solution that reached the lungs depending on the injection volume $(P<0.001)$, but not depending on animal age.

Conclusions: In this first report on quantitative imaging by PET and CT in small animals, we confirmed the suitability of the transnasal route with an instilled volume of $30 \mu \mathrm{l}$ delivering fluids into the lower airways, although only about $40 \%$ of the dose reaches the lungs.
\end{abstract}

Key words: PET, FDG, CT, Transnasal administration, Bronchial asthma

\section{Introduction}

Alergic bronchial asthma is a chronic inflammatory A disease with an increasing worldwide prevalence.

L Conejero was with the Hospital General Universitario Gregorio Marañón. She is now with the Immunology Unit, Department of Infectious and Tropical Diseases, London School of Hygiene \& Tropical Medicine, London, UK.

Correspondence to: M Desco; e-mail: desco@mce.hggm.es
Current pharmacologic therapies provide nonspecific shortterm relief from symptoms, but the efficacy of etiological treatments based on allergen immunotherapy is still limited. Nowadays, many asthma studies focus on reducing morbidity. However, ethical restrictions on human studies make murine models particularly useful, since it has been demonstrated that the allergic response in mouse lungs closely resembles certain aspects of human asthma [3].

Experimental protocols for airway allergen challenge in these models of airway disease involve three main routes of administration: (1) transnasal [4], (2) intratracheal [7], and 
(3) inhaled [11, 12, 18]. Our study focuses on transnasal administration. Although the procedure has been widely used, neither the amount of allergen that reaches the lungs nor the distribution within the airways has been well established.

Positron emission tomography (PET) is a noninvasive imaging technique that allows for reliable measurement of the concentration of radiotracers in the body of a living subject. Recent advances in detector design have enabled the construction of high-resolution, small-animal PET scanners for imaging small laboratory animals, thus opening new areas of research on the brain, tumors, preclinical evaluation of new radiopharmaceuticals, as well as gene expression and gene therapy $[15,17]$.

Computed tomography (CT) is also a noninvasive imaging technique that uses $\mathrm{X}$-rays to provide anatomical information. Image contrast depends on the attenuation coefficient of the X-rays and is directly correlated to tissue density [15]. CT has proven to be a useful complementary tool when combined with other modalities of functional imaging, since it provides structural information that makes it possible to accurately locate activity distribution within the body of a living animal.

We used PET and CT scanners to assess both the anatomical distribution and the amount of solution which reached the lungs immediately after transnasal delivery.

\section{Materials and Methods}

\section{Animals and Experimental Protocol}

Forty six female BALB/c mice were used in the experiment. Animals were purchased from IFFA CREDO SA (Barcelona, Spain), maintained at a constant temperature $\left(24 \pm 0.5^{\circ} \mathrm{C}\right)$ under a $12 \mathrm{~h} \mathrm{light/dark} \mathrm{cycle,} \mathrm{and} \mathrm{permitted} \mathrm{free} \mathrm{access} \mathrm{to} \mathrm{commercial}$ rodent laboratory chow and water. The following three groups were established based on volume administered and age:

(a) Group A (N 10): $15 \mu$ l administered to 8 to 10 week old mice;

(b) Group B (N 20): $30 \mu \mathrm{l}$ administered to 8 to 10 week old mice; and

(c) Group C ( $N$ 16): $30 \mu$ ladministered to 32 week old mice.

Once mice were anesthetized with inhaled Forane ${ }^{\circledR}$ (Abbott Laboratories Ltd., Kent, UK), they were placed upright and received the positron emitting tracer 2 deoxy $2\left[{ }^{18} \mathrm{~F}\right]$ fluoro D glu cose $\left({ }^{18} \mathrm{FDG}\right)$ transnasally. The tracer was dissolved in $15 \mu \mathrm{l}(8.18 \pm$ $1.77 \mathrm{MBq})$ or $30 \mu \mathrm{l}(11.98 \pm 3.69 \mathrm{MBq})$ of saline. Animals were administered half the volume through each nostril with a micropipette and were kept upright for $\sim 30 \mathrm{~s}$ to facilitate the access of the tracer to the airway. One minute after the instillation, the mice were anesthetized with isofluorane $(5 \%$ for induction and $2 \%$ for maintenance in $100 \% \mathrm{O}_{2}$ ) and were introduced in the PET scanner in head in prone position. The mice were dynamically scanned for $320 \mathrm{~s}$, generating a study with eight frames of $40 \mathrm{~s}$ each. Moreover, ten mice from group B were also scanned using a static whole body imaging protocol of $320 \mathrm{~s}$ with two bed positions and one frame to check that the solution did not enter compart ments other than the lungs or upper airways.

All experimental animal procedures were performed in compli ance with the European Communities Council Directive of 24 November 1986 (86/609/EEC) and were approved by the Institu tional Animal Care and Use Committee of the Hospital.

\section{PET Imaging}

PET scans were performed with a small animal PET scanner (rPET SUINSA Medical Systems, Madrid, Spain) [15], and acquisitions were centered on the thoracic region. Tomographic images were reconstructed using a 3D FBP (filtered back projection) algorithm that produced 55 slices measuring $55 \times 55$ pixels each. In the case of whole body imaging protocol, the study had 102 slices of $55 \times$ 55 pixels. The voxel size was $0.81 \times 0.81 \times 0.81 \mathrm{~mm}^{3}$, and the spatial resolution in these images was $1.65 \mathrm{~mm}$ full width at half maximum isotropic. The energy window was $400700 \mathrm{keV}$, and decay, deadtime, randoms, and scatter corrections were applied. Attenuation correction was not performed since the attenuation correction factor for mouse lungs should be close to 1 due to the very low amount of tissue present on the thoracic tomographic slices. Previous studies [19] have demonstrated that it is possible to perform accurate quantification of ${ }^{18}$ FDG PET images of small mice without attenuation correction even in more dense tissues as the liver. Therefore, we assume that attenuation correction would not significantly change the quantitative results.

\section{Analysis of PET Data}

To obtain quantitative data of lung activity, three regions of interest (ROI) were drawn on coronal, sagittal, and transaxial sections of the dynamic studies: right lung, left lung, and total field of view (FOV) activity. Time activity curves were obtained and extrap olations to time 0 were calculated by fitting the data to an exponential function. These values at time 0 were used to estimate the initial distribution, specifically the fraction of the activity going to the lungs with respect to the injected dose (represented by the total FOV activity). Results are thus presented as a percentage of total FOV activity. Figure 1 shows an example of these time activity curves in a mouse instilled with $30 \mu 1$.

\section{CT Study}

Those animals scanned using a PET whole body imaging protocol were also scanned on a small animal CT scanner [16]. CT images covered the animal from nose to abdomen and were reconstructed using a modified Feldkamp, Davis, and Kress algorithm. The voxel size was $0.25 \times 0.25 \times 0.25 \mathrm{~mm}^{3}$. Tube current and amperage $(50 \mathrm{kV}, 200 \mu \mathrm{A})$ were optimized for lung imaging.

The tracheal volume in a subgroup of eight animals from group $\mathrm{B}$ was obtained from the CT images by drawing on coronal sections an ROI corresponding to the tracheal lumen.

CT and PET images were co registered using a marker based rigid registration method to obtain an initial realignment, followed by a refinement step based on an automatic mutual information registration algorithm $[2,10]$. 

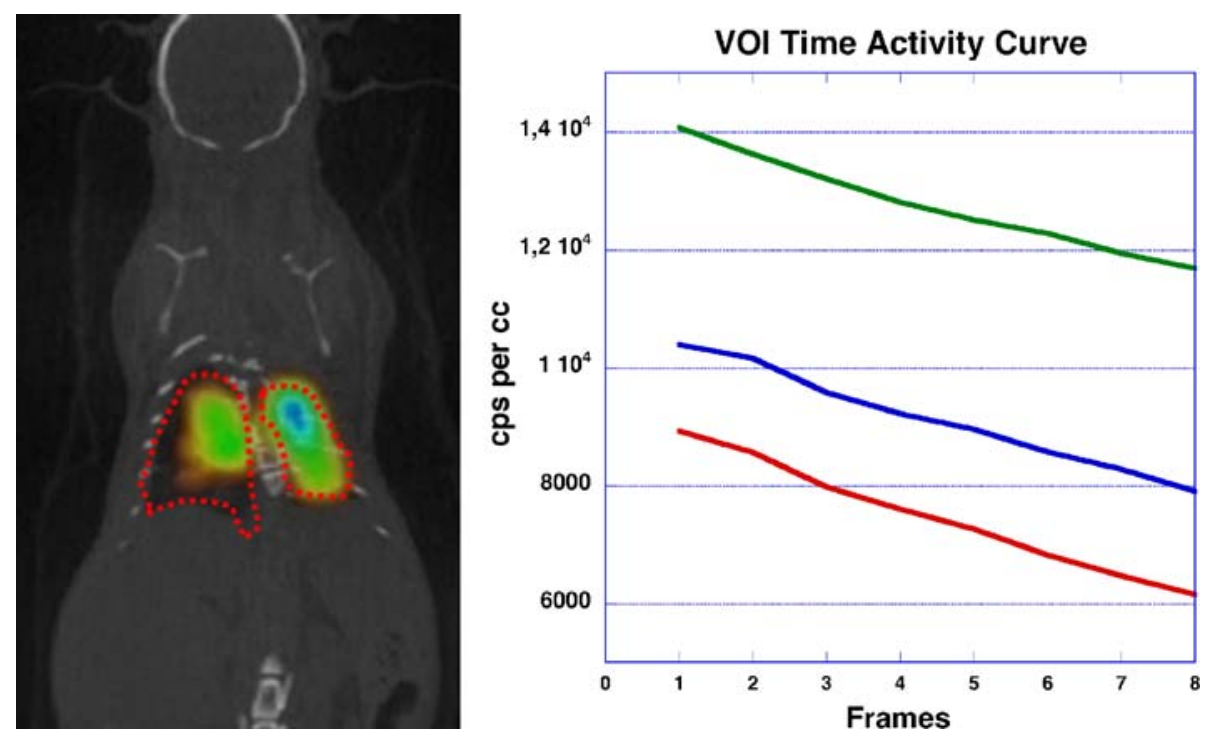

Fig. 1. Left panel, Left lung and right lung activity are measured by 3D segmentation of the lungs on the CT image. Right panel, dynamic curves of tracer distribution in a mouse instilled with $30 \mu \mathrm{l}$ of FDG, obtained at 40 s/frame (green line, total FOV activity; blue line, right lung; and red line, left lung.

\section{Statistical Analysis}

ROI data were analyzed by means of one way analysis of variance (ANOVA) followed by post hoc tests (Scheffé). Hypothesis of normality of distributions and homogeneity of variance were assessed using the Kolmogorov Smirnov and Levene tests, respectively.

\section{Results}

An average of $5.69 \pm 4.51 \%$ (mean $\pm \mathrm{SD}$ ) of the administered solution reached the lungs in group A, $41.84 \pm 8.03 \%$ in group $B$ and $36.65 \pm 16.15 \%$ in group C. All the studies included the animal head well inside the field of view. The proportion of solution delivered to each lung in the different groups is summarized in Table 1.

ANOVA revealed a significant difference between groups in the total proportion of administered solution that reached the lungs, depending on injection volume $(P<0.001)$, but not on animal age.

No significant differences in the distribution of the solution were observed between the left and right lungs in any of the groups.

The percentage of solution reaching the lungs showed very low values $(<4 \%)$ in four animals from group A. Figure 2 shows projection images from two mice immediately after administering the tracer solution (FDG). The solution was properly instilled in the case showed in panel a, but not in the case showed in panel $b$.

The tracheal volume of mice $8-10$ weeks old was $13.6 \pm$ $1.8 \mu 1$.

The co-registered PET and CT studies of mice scanned using a whole body imaging position protocol confirmed the anatomical location of the tracer after transnasal administration. Activity was detected in lungs, trachea, and upper airways (nose, throat), but not in the gastrointestinal tract (Fig. 3). Figure 4 shows combined PET-CT axial sections of a representative mouse after transnasal administration of $30 \mu \mathrm{l}$ containing approximately $300 \mu \mathrm{Ci}$.

\section{Discussion}

The study of in vivo animal models has provided valuable information on several aspects of the pathogenesis and treatment of allergy and asthma. Murine models of allergic asthma have usually been generated by inducing systemic sensitization followed by an airway challenge using nebulized or transnasal allergen $[4,20]$. In the present study, we evaluated the amount of solution reaching the lung and its distribution within the airways after a transnasal challenge by ${ }^{18}$ FDG by PET and CT imaging.

Table 1. Percentage of solution delivered to each lung in the different groups of mice

\begin{tabular}{|c|c|c|c|}
\hline & Right Lung & Left Lung & Both Lungs \\
\hline Group A (N 10) $15 \mu 1,810$ weeks old & $2.63 \pm 2.72$ & $3.06 \pm 2.07$ & $5.69 \pm 4.51$ \\
\hline Group B $(N \quad 20) 30 \mu 1,810$ week old & $25.21 \pm 7.25^{*}$ & $16.62 \pm 3.66^{*}$ & $41.84 \pm 8.03 *$ \\
\hline Group C $(N \quad 16) 30 \mu 1,32$ week old & $17.91 \pm 7.87^{*}$ & $18.74 \pm 12.28^{*}$ & $36.65 \pm 16.15^{*}$ \\
\hline
\end{tabular}

$N$ represents the number of animals in each group. Values are expressed as mean percentage \pm SD

$* P<0.001$ versus group A 


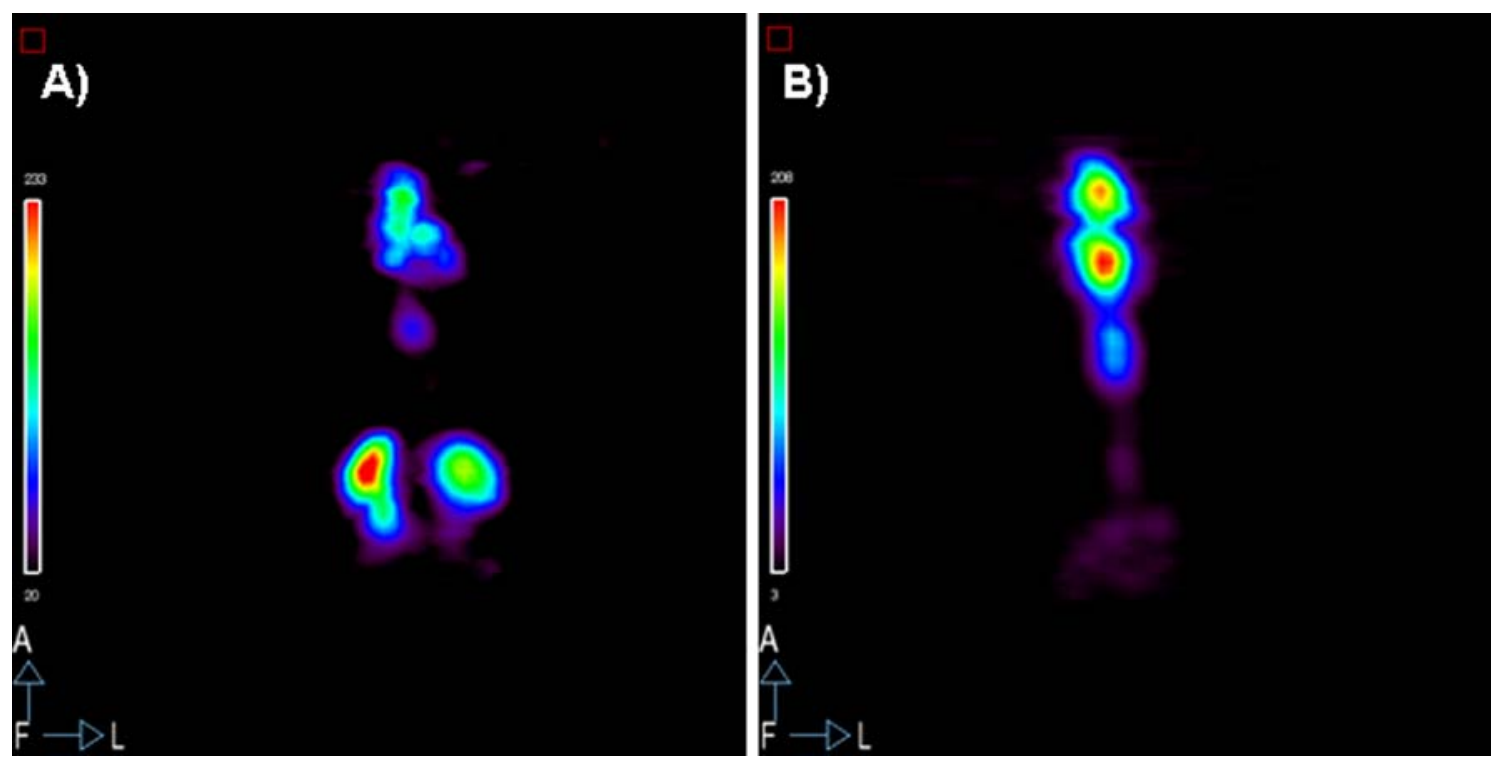

Fig. 2. Projection images from two mice immediately after administering the tracer solution (FDG). a Coronal view of a properly instilled animal (head is up, the two bright blobs in the middle are the lungs). b Coronal view of a bad instillation (head is up; there is almost none activity at lung level).

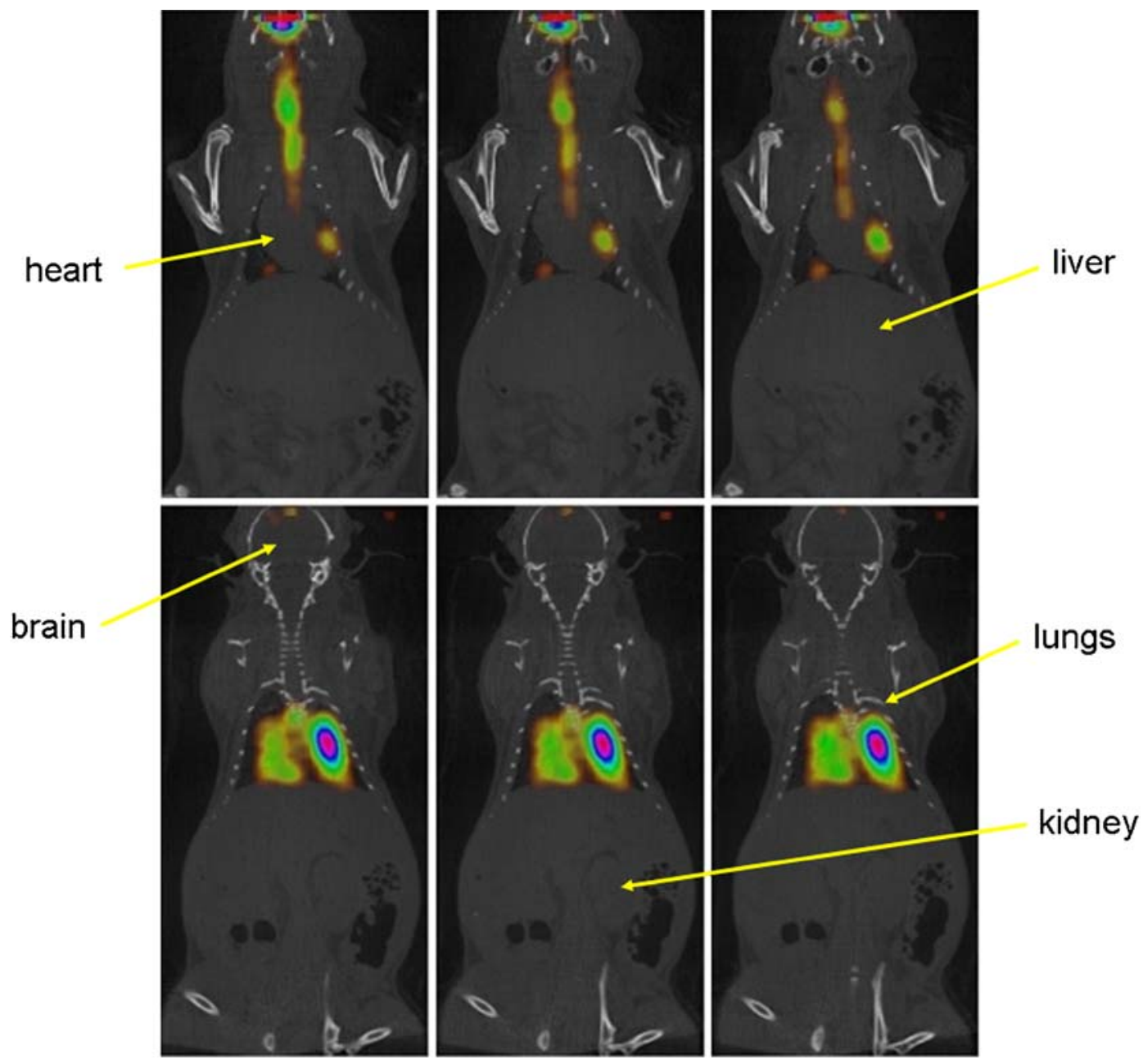

Fig. 3. Co-registered PET and CT coronal slices at the first time frame. From top left to bottom right, the slices go from ventral to dorsal. The larynx is clearly visible on the more ventral slices (upper pane/). 

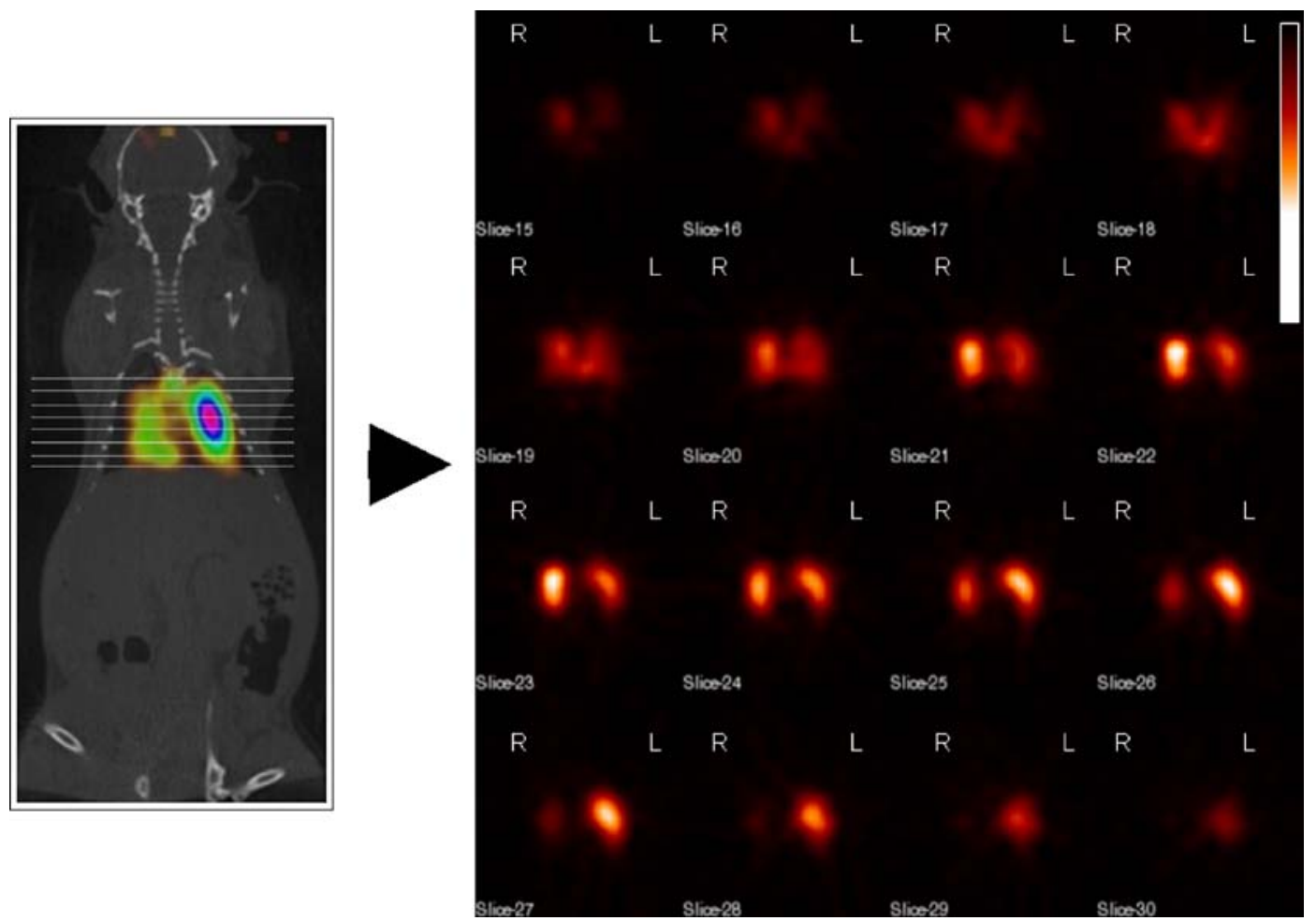

Fig. 4. Images from a representative mouse after transnasal administration of $30 \mu$ l containing 11.1 MBq of FDG. Left panel, Co-registered PET and CT studies superimposed. Right panel, Axial sections of the lungs in the PET study.

In this study, we used an aqueous solution of FDG to examine the anatomical distribution of the dose after intranasal administration. The activity of FDG decreased with time, as shown in Fig. 1, probably due to the diffusion of FDG into the bloodstream and posterior uptake by different tissues. The acquisition duration of our study (about $5 \mathrm{~min}$ ) was too short to see clear FDG uptake in any specific organ. The study of this diffusion and redistribution process is not of interest in this work; therefore, we used time $=0$ extrapolated values to estimate the initial dose distribution.

In our work, we assume that immediately after the administration of FDG, all the dose remains in the FOV, which covers the upper respiratory tract, lungs, esophagus, and stomach. In this regard, any other tracer could have been used instead of FDG, as any specific metabolization of the radiopharmaceutical is irrelevant at time $=0$ when the initial distribution only depends on physical mechanisms.

The influence of the age of the mice on the tracer distribution was also evaluated. Mice are born without alveoli, and these are formed rapidly within the first few postnatal weeks [1]. The development of the alveoli and the elastic fiber is complete by 38 days of age [8]. The present study was performed on animals with fully developed alveoli. We studied animals that were 8 to 10 weeks old (young adults) and 32 weeks old (old adults). Young adults are usually used for acute asthma studies and old adults are used in chronic asthmatic models. We did not detect any age-related differences in tracer distribution in the lungs of the animals that received $30 \mu \mathrm{l}$, indicating that transnasal challenge may be valuable in both acute and chronic models.

Efficiency of dose delivery to the lung can be defined as the percentage of the dose deposited in the lungs $(\mathrm{dl})$ relative to the dose delivered (dd) [(Table 1; Efficiency $=100 \times \mathrm{dl} /$ dd)]. After administering the allergen transnasally with the animal supine and its neck hyperextended, Tomaki et al. [13] reported that $31 \%$ of administered particles finished in the lower airways. In this study, the efficiency of transnasal administration was examined using Evans blue dye and isolating tissue from the trachea, bronchi, lungs, esophagus, stomach, and nose. Our study is the first to use an in vivo molecular imaging technique (PET) to evaluate the efficiency of transnasal challenge in mice, and we obtained an overall value in the lungs close to $40 \%$ of the delivered dose. The regional distribution of FDG in the lungs was apparently homogenous, but no attempt was made to perform a quantitative assessment.

The optimum volume instilled for challenge is a controversial issue. In some protocols, the instilled volumes ranged from $50 \mu 1[5,9,12]$ to $100 \mu 1$ over several days [6]. Other authors use only $10 \mu \mathrm{l}$ over several days [14]. We compared the effect of two different volumes of instillation, 15 and $30 \mu \mathrm{l}$, and found significant differences between them: The animals that received $15 \mu 1$ showed approximately $35 \%$ less activity in the lungs than those that received $30 \mu \mathrm{l}$. These results agree with the data shown by Tomaki et al. [13] who reported that $31 \%$ of the dose went into the 
lungs with $25 \mu 1$. In our case, when we administered $30 \mu 1$, the amount of tracer in the lungs reached about $40 \%$. We obtained a tracheal volume of about $13.6 \pm 1.8 \mu 1$ in mice aged $8-10$ weeks. This volume could explain the approximately sixfold difference in mean lung distribution between animals from group $\mathrm{A}$ and groups $\mathrm{B} / \mathrm{C}$, as it implies that a minimum volume of $30 \mu \mathrm{l}$ may be necessary in order to ensure that at least $40 \%$ of the administered dose actually reaches the lungs. This result is interesting, since it is necessary to find a compromise between the volume administered (trying not to drown the animal) and the amount of allergen/medication that reaches the lungs. We had previously tried administering $50 \mu \mathrm{l}$, but some mice died (data not shown). In fact, we have demonstrated that the instillation of allergen in a volume of $30 \mu \mathrm{l}$ can induce lung inflammation in airway disease models at a lower risk of drowning [4].

In those cases of group A where the dose reaching the lungs was very low, most of the FDG solution appeared at the upper nasal passages and esophagus. This probably means that either the positioning for instillation, the instillation duration, or the instilled volume was not technically adequate. In this way, the large variation within and between groups may reflect the technical difficulty of the nasal administration, which sometimes even leads to a totally incorrect dose delivery, particularly when the instilled volume is small, as is the case with group A. According to this interpretation, a proper instillation technique may constitute a key point of the procedure, and a number of failures in the administration have to be accepted as a possibility by the experimenters.

Our study showed the advantage of using multimodality image registration (PET and CT studies). PET allowed us to quantify the distribution of the tracer administered transnasally in the upper airways (nose, throat), trachea, and the lungs (Fig. 3). CT images provide anatomical information that, co-registered with PET, enabled us to demonstrate that the solution reaches the anatomical target in a sufficient amount.

One possible limitation of our study derives from the extrapolation of time-activity curves to time $=0$ by fitting data to an exponential function. We chose an exponential function because of its good fit to our data and because it is a simple and robust model. We did not test whether another function could yield more precise results.

We believe that the information provided by these results may be of interest for researchers working in the field of airway allergen challenge, as there is no available data on the amount of allergen that may really reach the lung in in vivo studies. It may also be advisable for the researchers to be aware about the possibility of an incorrect instillation that may lead to a near-zero dose delivered to the lung in a small percentage of the cases.

In conclusion, in this first report on quantitative imaging by PET and CT in small animals, we confirmed that the transnasal route with an instilled volume of $30 \mu \mathrm{l}$ is suitable for delivering fluids into the lower airways, although only about $40 \%$ of the dose reaches the lungs.

Acknowledgments. This work was supported by grants from Ministerio de Ciencia y Tecnología (TEC2004-07052-C02-01), Ministerio de Sanidad y Consumo (CIBER CB06/01/0079 CIBER CB07/09/0031 and FIS 2001/ 0598), Ministerio de Industria (CDTEAM Project) and Fundación de Investigación Médica Mutua Madrileña.

\section{References}

1. Amy RW, Bowes D, Burri PH, Haines J et al (1977) Postnatal growth of the mouse lung. J Anat 124(Pt 1):131 151

2. Arun KS, Huang TS, Blostein SD (1987) Least-squares fitting of two 3D point sets. IEEE Trans Pattern Anal Machine Itell, PAMI 9(5):698 700

3. Campbell EM, Kunkel SL, Strieter RM, Lukacs NW (1998) Temporal role of chemokines in a murine model of cockroach allergen-induced airway hyperreactivity and eosinophilia. J Immunol 161(12):7047 7053

4. Conejero L, Higaki Y, Baeza ML, Fernandez M et al (2007) Polleninduced airway inflammation, hyper-responsiveness and apoptosis in a murine model of allergy. Clin Exp Allergy 37(3):331 338

5. Costa-Pinto FA, Basso AS, Russo M (2007) Role of mast cell degranulation in the neural correlates of the immediate allergic reaction in a murine model of asthma. Brain Behav Immun 21(6):783 790

6. Havaux X, Zeine A, Dits A, Denis O (2005) A new mouse model of lung allergy induced by the spores of Alternaria alternata and Cladosporium herbarum molds. Clin Exp Immunol 139(2):179 188

7. Hogaboam CM, Blease K, Mehrad B, Steinhauser ML et al (2000) Chronic airway hyperreactivity, goblet cell hyperplasia, and peribronchial fibrosis during allergic airway disease induced by Aspergillus fumigatus. Am J Pathol 156(2):723 732

8. Kawakami M, Paul JL, Thurlbeck WM (1984) The effect of age on lung structure in male BALB/cNNia inbred mice. Am J Anat 170(1):1 21

9. Lopez E, del Pozo V, Miguel T, Sastre B et al (2006) Inhibition of chronic airway inflammation and remodeling by galectin- 3 gene therapy in a murine model. J Immunol 176(3):943 950

10. Maes F, Collignon A, Vandermeulen D, Marchal G et al (1997) Multimodality image registration by maximization of mutual information. IEEE Trans Med Imaging 16(2):187 198

11. Sakai K, Yokoyama A, Kohno N, Hamada H et al (2001) Prolonged antigen exposure ameliorates airway inflammation but not remodeling in a mouse model of bronchial asthma. Int Arch Allergy Immunol 126(2):126 134

12. Shinagawa K, Kojima M (2003) Mouse model of airway remodeling: strain differences. Am J Respir Crit Care Med 168(8):959 967

13. Tomaki M, Zhao LL, Lundahl J, Sjostrand M et al (2000) Eosinophilopoiesis in a murine model of allergic airway eosinophilia: involvement of bone marrow IL-5 and IL-5 receptor alpha. J Immunol 165 (7):4040 4050

14. Vanoirbeek JA, Tarkowski M, Vanhooren HM, De Vooght V et al (2006) Validation of a mouse model of chemical-induced asthma using trimellitic anhydride, a respiratory sensitizer, and dinitrochlorobenzene, a dermal sensitizer. J Allergy Clin Immunol 117(5):1090 1097

15. Vaquero JJ, Lage E, Redondo S, Abella M et al (2005) Co-planar PET/ $\mathrm{CT}$ for small animal imaging. IEEE Nuclear Science Symposium Conference Record (CD-ROM), pp 17481751

16. Vaquero JJ, Redondo S, Lage E, Abella M et al (2008) Assessment of a new high-performance small-animal X-ray tomograph. IEEE T Nucl Sci 55(3):898 905

17. Wang Y, Seidel J, Tsui BMW, Vaquero JJ et al (2006) Performance evaluation of the GE healthcare explore vista dual-ring small-animal pet scanner. J Nucl Med 47:1891 1900

18. Yiamouyiannis CA, Schramm CM, Puddington L, Stengel $\mathrm{P}$ et al (1999) Shifts in lung lymphocyte profiles correlate with the sequential development of acute allergic and chronic tolerant stages in a murine asthma model. Am J Pathol 154(6):1911 1921

19. Zingone A, Seidel J, Aloj L, Caraco C et al (2002) Monitoring the correction of glycogen storage disease type 1a in a mouse model using [(18)F]FDG and a dedicated animal scanner. Life Sci 71(11):1293 1301

20. Zosky GR, Sly PD (2007) Animal models of asthma. Clin Exp Allergy 37(7):973 88 